\title{
Piezoelectric Wind Energy Harvesting from Self-Excited Vibration of Square Cylinder
}

\author{
Junlei Wang, ${ }^{1,2,3}$ Sheng Wen, ${ }^{4}$ Xingqiang Zhao, ${ }^{2}$ Min Zhang, ${ }^{5,6}$ and Jingyu $\operatorname{Ran}^{3,7}$ \\ ${ }^{1}$ College of Chemical Engineering and Energy, Zhengzhou University, Zhengzhou, Henan 450002, China \\ ${ }^{2}$ Jiangsu Engineering Research Center on Meteorological Energy Using and Control, Nanjing University of \\ Information Science \& Technology, Nanjing 210000, China \\ ${ }^{3}$ Key Laboratory of Low-Grade Energy Utilization Technologies and Systems, Chongqing University, \\ Ministry of Education of China, Chongqing 400044, China \\ ${ }^{4}$ College of Engineering, South China Agricultural University, Guangzhou, Guangdong 510642, China \\ ${ }^{5}$ School of Mechanical Engineering, Shanghai Jiao Tong University, Shanghai 200240, China \\ ${ }^{6}$ Shipping and Marine Engineering College, Chongqing Jiao Tong University, Chongqing 40074, China \\ ${ }^{7}$ Power Engineering School, Chongqing University, Chongqing 400044, China
}

Correspondence should be addressed to Sheng Wen; jlwang@zzu.edu.cn

Received 4 January 2016; Revised 27 April 2016; Accepted 8 May 2016

Academic Editor: Chengkuo Lee

Copyright (C) 2016 Junlei Wang et al. This is an open access article distributed under the Creative Commons Attribution License, which permits unrestricted use, distribution, and reproduction in any medium, provided the original work is properly cited.

Self-excited vibration of a square cylinder has been considered as an effective way in harvesting piezoelectric wind energy. In present work, both of the vortex-induced vibration and unstable galloping phenomenon process are investigated in a reduced velocity $\left(U_{r}=U / \omega_{n} \cdot D\right)$ range of $4 \leq U_{r} \leq 20$ with load resistance ranging in $100 \Omega \leq R \leq 1 \mathrm{M} \Omega$. The vortex-induced vibration covers presynchronization, synchronization, and postsynchronization branches. An aeroelectromechanical model is given to describe the coupling of the dynamic equation of the fluid-structure interaction and the equation of Gauss law. The effects of load resistance are investigated in both the open-circuit and close-circuit system by a linear analysis, which covers the parameters of the transverse displacement, aerodynamic force, output voltage, and harvested power utilized to measure the efficiency of the system. The highest level of the transverse displacement and the maximum value of harvested power of synchronization branch during the vortexinduced vibration and galloping are obtained. The results show that the large-amplitude galloping at high wind speeds can generate energy. Additionally, energy can be harvested by utilization of the lock-in phenomenon of vortex-induced vibration under low wind speed.

\section{Introduction}

Wireless sensor network (WSN) has been widely used in various fields, such as environmental monitoring, medicine, and military. The bottleneck of WSN lies in the limited battery lifetime. The frequent battery replacement and charging are time-consuming, expensive, and environment unfriendly. An interest in powering the sensors in a self-sustaining way has been increased. In literatures, the detailed information of various micropower generators harvesting energy from wind, human motions, light, and temperature difference or the other ways can be found [1-6].
Self-excited vibration (SEV) is a common phenomenon in nature and engineering. Blevins [7] investigated the mechanism of the self-excited vibration, as shown in Figure 1.

In $\mathrm{SEV}$, when vortex shedding frequency gets close to the natural frequency of bluff body, the phenomenon called "synchronization" or "lock-in" occurs and the oscillating amplitude can reach a high level. Meanwhile, for a bluff body with square-section under high Reynolds numbers, when wind speed exceeds a critical value, the system begins to undergo aerodynamic instability and the phenomenon called galloping occurs. To utilize the two phenomena for harvesting flow energy, some devices have been developed to 
Forward information

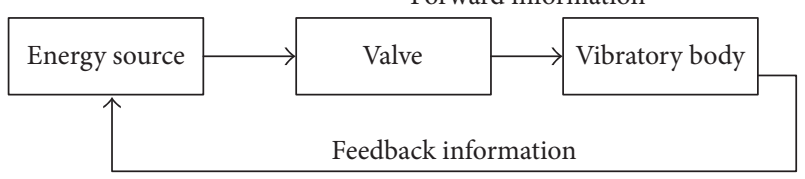

FIGURE 1: Feedback mechanism of self-excited vibration.

produce energy from structural vibrations induced by wind or water flow.

Taylor et al. [8] and Allen and Smits [9] described an "eel" equipment and investigated the utilization of flexible and flaglike PVDF (polyvinylidene fluoride) sheets to harvest power in a water channel. Robbins et al. [10] investigated a similar method for utilizing PVDF sheet to generate power in a wind tunnel.

At Marine Renewable Energy Laboratory (MRELab) of University of Michigan, Raghavan and Bernitsas [11] and Lee and Bernitsas [12] have developed an ocean energy harvesting system called VIVACE (Vortex-Induced Vibration for Aquatic Clean Energy). The device harvests energy from the oscillations induced by vortex shedding from circular cylinders. The factors, such as mass ratio, mechanical damping, Reynolds numbers, and aspect ratio (length to diameter) of the cylinder, were investigated. Wu et al. [13] provided a CFD code to solve VIV problem of a single cylinder with PTC (passive turbulence control). In addition, a CFD code has been developed by Ding et al. [14] in OpenFOAM to solve VIV of multiple circular cylinders.

At University of Virginia, Mehmood et al. [15] and Abdelkefi et al. [16] developed a method for extracting flow energy by using a wake oscillator model and direct numerical simulation method. It was reported that the presence of the hardening behavior was due to aerodynamic nonlinearity. They also explained the decisive effects of the load resistance on the synchronization region and level of the power harvesting. The maximum level of the power harvesting could appear simultaneously with the minimum value in the transverse displacement due to a shunt damping effect. Besides, Abdelkefi and his coauthors $[17,18]$ investigated the potential utility of harvesting energy from base excitations with VIV or galloping. Barrero-Gil et al. [19] theoretically investigated the feasibility of energy harvesting from galloping structures. They used a cubic polynomial to represent the aerodynamic characteristics and obtained an expression for the harnessing of the flow energy. To investigate the possibility of harvesting energy from other flow-induced vibrations, various researches have been performed [20-22]. For extracting energy from flutter phenomenon, Abdelkefi and Nuhait [20] discussed the effects of a cambered wingbased piezoaeroelastic energy harvester. Both linear and nonlinear mathematical models have been developed to predict the performance of the device.

It is worth noting that both vortex-induced vibration and galloping of a square-section structure can be utilized in the same piezoelectric wind energy harvesting equipment, because both of the two phenomena can cause large oscillating amplitude that can be good power source to excite
TABLE 1: Properties of electromechanical system.

\begin{tabular}{llc}
\hline Symbol & Property & Value \\
\hline$M$ & Mass of the cylinder per unit length & $0.44 \mathrm{~kg}$ \\
$K$ & Stiffness per unit length & $854 \mathrm{~N} / \mathrm{m}$ \\
$C$ & Damping per unit length & $19.2 \mathrm{Nm} / \mathrm{s}$ \\
$D$ & Side length of the cylinder & $1.5 \mathrm{~cm}$ \\
$\xi$ & Damping ratio & 0.0013 \\
$f_{n}$ & Natural frequency & $7.006 \mathrm{~Hz}$ \\
$c_{p}$ & Capacitance & $120 \mathrm{nF}$ \\
$\theta$ & Electromechanical coupling & $1.55 E-3 \mathrm{~N} / \mathrm{V}$ \\
\hline
\end{tabular}

the piezoelectric device to vibrate. However, there are few literatures focusing on considering of the two phenomena in the same energy harvesting system.

This paper describes a piezoelectric device considering both vortex-induced vibration and galloping of a squaresection cylinder for harvesting wind energy. In present work, a two-dimensional dynamical system is used to investigate the coupling of outflow field, cylinder, and the piezoelectric transducer. The parameters in current numerical calculation system are listed in Table 1.

The aeroelectromechanical coupled governing equations are solved to determine the level of harvested energy from SEV. The harvested power is investigated under different operating conditions including the reduced wind velocities and load resistance. In Section 2, the physical and mathematical models are discussed, and validation of the fluid flow solver and the coupling scheme with previous experimental data is presented. In Section 3, the open-circuit system with infinite load resistance is discussed. In this part, a simplified model is introduced to calculate the open-circuit voltage output. In Section 4, the close-circuit system with different load resistance ranging from $100 \Omega$ to $1 E 6 \Omega$ is discussed; the effects of reduced velocity and load resistance on the oscillation amplitudes, fluctuating lift coefficient, voltage output, and harvested power are studied. In Section 5, the conclusions are presented.

\section{Physical and Mathematical Models}

2.1. Physical Model of SEV. A simplified SEV-based energy harvester configuration was explored. The model consists of a spring-mounted square-section cylinder and a piezoelectric transducer attached to its transverse degree of freedom. The cylinder vibrates freely under the action of an incoming flow in the transverse direction which is shown in Figure 2. The system has a mass per unit length $M$, a spring-coefficient $K$, damping $C$, and an electric resistance $R$. The equation governing the mechanical system is

$$
M\left(\ddot{y}+2 \xi \omega_{n} \dot{y}+\omega_{n}{ }^{2} y\right)=F_{y}=\frac{1}{2} \rho U^{2} D C_{y},
$$

where $F_{y}$ is the fluid force per unit length in the normal direction to the incoming flow, the dot symbol is differentiation with respect to time $t, \rho$ is the density of the incoming flow, $U$ is the velocity of the incident flow, $D$ is the characteristic dimension of the body normal to the flow, and $C_{y}$ is 


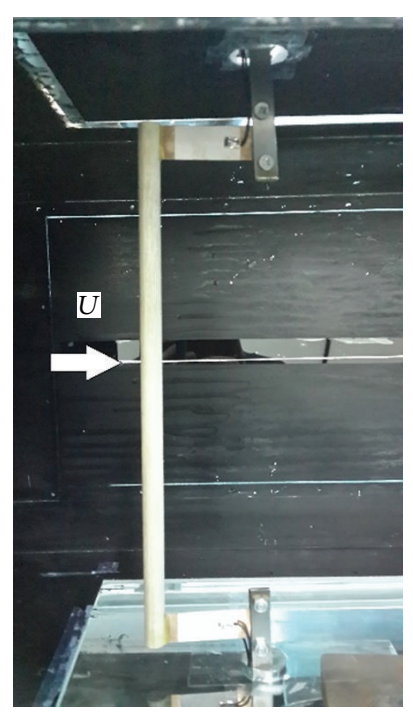

(a)

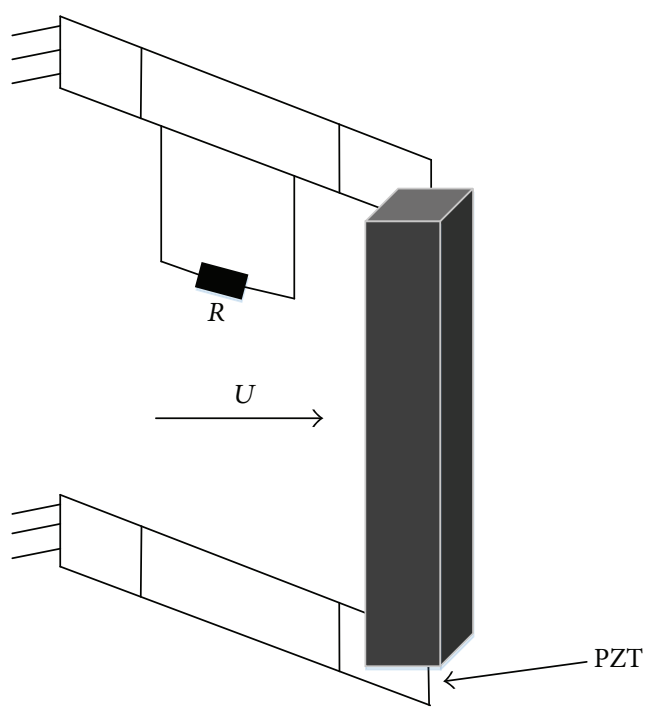

(b)

FIGURE 2: (a) The experimental setup of the vibrational system. (b) Schematic of the proposed square-cylinder based piezoaeroelastic energy harvester.

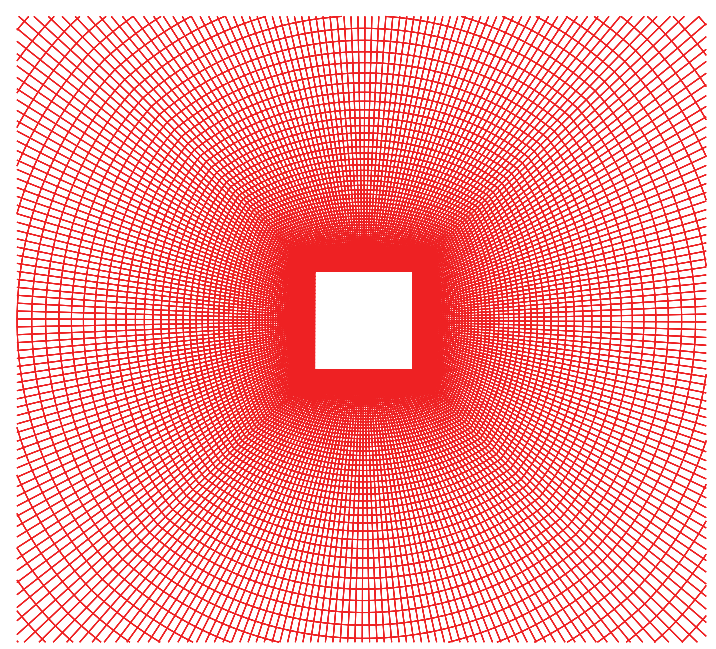

(a)

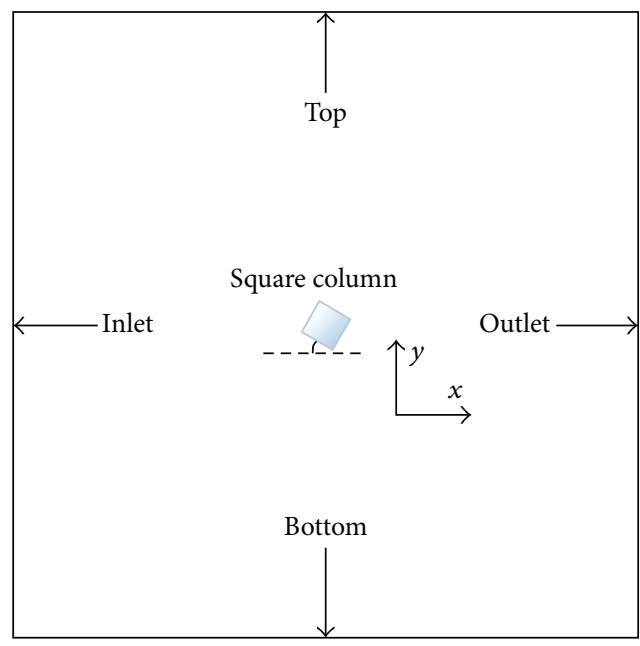

(b)

Figure 3: (a) The computational grid in present work. (b) The boundary conditions of the system.

the instantaneous fluid force coefficient in the transverse direction to the incident flow. For galloping, particularly, a cubic polynomial can be used to approximate the vertical fluid force coefficient $C_{y}$ [23]:

$$
C_{y}=\left[a_{1} \frac{\dot{y}}{U}+a_{3}\left(\frac{\dot{y}}{U}\right)^{3}\right]
$$

where $a_{1}$ and $a_{3}$ are empirical coefficients measured in static tests. It is noted that if the cross-section of the structure is symmetric about a line in the flow direction through the center of the section, only odd harmonics $a_{1}, a_{3}$, and so forth in the series are nonzero [7].
The relationship between the parameters of the $M-C-K$ system can be presented as

$$
\begin{aligned}
& K=\omega_{n}{ }^{2} \cdot M, \\
& C=2 \xi \cdot M \cdot \omega_{n},
\end{aligned}
$$

where $\omega_{n}$ is the natural frequency of oscillations and $\xi$ is the damping ratio.

For the axial symmetry of the system, a two-dimensional model was used to describe the energy harvesting system which was showed in Figure 2. Figure 2(a) presents the real vibrational system. The structured grid is used for its computational stability to solve the moving grid problem and the near wall grid is shown in Figure 3(a). The cylinder undergoes 
SEV when the harvester is subjected to an incoming flow; Figure 3(b) shows the boundary condition of the system. If the free stream velocity comes to a critical value of the onset of "synchronization," the vortex shedding frequency can be locked into the cylinder oscillating frequency; the amplitude can become pretty high. If the flow velocity exceeds the second critical value, galloping occurs in the transverse direction, and higher level amplitude can be obtained.

2.2. Electromechanical Coupling Model. The fluid-electromechanical coupling procedure for the $M-C-K$ system was briefly introduced by deriving the mathematical model of the system. The continuity and Navier-Stokes (N-S) equations and the one-dimensional constitutive equations of the piezoelectric transducer are given by

$$
\begin{aligned}
\frac{D \rho}{D t}+\rho S_{k k} & =0, \\
\frac{\rho D V_{i}}{D t} & =-\frac{\partial p}{\partial x_{i}}+\frac{\partial \tau_{i j}}{\partial x_{j}}, \\
\frac{\rho D}{D t} & =-\frac{\partial}{\partial t}+\frac{V_{k} \partial x}{\partial x_{k}} .
\end{aligned}
$$

Equation (6) is the total derivative operator, $p$ is the pressure, $\rho$ is the fluid density, $V_{i}$ is fluid velocity vector, $\tau_{i j}$ is the stress tensor, and $S_{k k}$ is the strain rate tensor. A nonslip boundary condition on the surface of a moving body is given as

$$
V(x, y)=V_{\Omega}(x, y), \quad x, y \in \Omega,
$$

where $\Omega$ is the wet surface of the deforming structure, $V_{\Omega}$ is moving velocity of $\Omega$, during the fluid-structure interaction process, and the cylinder moves up and down in the flow field under the boundary conditions, while the pressure $P_{\Omega}$ is acting on $\Omega$ and the forcing term $F_{y}$ in the structural equation shown in the following is generated.

In present work, we focused on the effect of load resistance $R$ on the energy harvesting system. Two different systems, open-circuit system and close-circuit system, were identified through the value of investigated load resistance $R$.

2.2.1. Electromechanical Coupling Model of the Open-Circuit System. For the single-degree-of-freedom (SDOF) system with a resistive load $(R),(1)$ can be rewritten with an additional electromechanical term and given by

$$
\begin{aligned}
M \ddot{y}+C \dot{y}+K y-\theta V & =F_{y}, \\
\theta \dot{y}+c_{p} \dot{V}+\frac{V}{R} & =0 .
\end{aligned}
$$

Or

$$
\theta \dot{y}+c_{p} \dot{V}=-\frac{V}{R}=I .
$$

For open-circuit condition, the equations of the SDOF coupling system with a resistive load $(R)$ can be rewritten as

$$
M \ddot{y}+C \dot{y}+K y-\theta V_{\mathrm{oc}}=F_{y},
$$

where $V_{\mathrm{oc}}$ is the open-circuit voltage and $I$ is the current. In this case if we set $R=\infty$, (10) can be available

$$
y=-\frac{c_{p}}{\theta} V_{\mathrm{oc}}
$$

Equation (12) can be added into (8) as

$$
M \ddot{y}+C \dot{y}+\left(k^{\prime}+\frac{\theta^{2}}{c_{p}}\right) y=F_{y} .
$$

The parameter $k^{\prime}=\omega_{n}{ }^{2} m+\theta^{2} / c_{p}$ is the coupling mechanical stiffness. Substituting (3) into (13) and introducing dimensionless variables $h=y / D$ and $\tau=\omega_{n} t$, a new equation form can be given as

$$
\ddot{h}+2 \xi \dot{h}+\left(\omega_{n}{ }^{2}+\frac{\theta^{2}}{c_{p} m}\right) h=\frac{\rho U^{2}}{2 m} C_{y} .
$$

Substituting (14) into (13) and introducing another twodimensionless mass ratio $m^{*}=m / \rho D^{2}$ and reduced velocity $U_{r}=U /\left(\omega_{n} D\right)$

$$
\begin{aligned}
\ddot{h}+ & 2 \xi \dot{h}+\left(\omega_{n}^{2}+\frac{\theta^{2}}{c_{p} m^{*}}\right) h \\
& =\frac{U^{* 2}}{2 m^{*}}\left(a_{1} \frac{\dot{h}}{U_{r}}+a_{3}\left(\frac{\dot{h}}{U_{r}}\right)^{3}\right) .
\end{aligned}
$$

Equation (15) expresses the dimensionless form of electromechanical coupling. For low Reynolds numbers, the coefficients $a_{1}$ and $a_{3}$ can be given as

$$
\begin{aligned}
& a_{1}=-2.7+0.017 \mathrm{Re}, \\
& a_{3}=10-0.096 \mathrm{Re}-0.001 \mathrm{Re}^{2} .
\end{aligned}
$$

In cases of $\mathrm{Re}>200$, the linear and nonlinear coefficients can be given by Parkinson and Smith's experimental results $[24,25]$, where $a_{1}=2.3$ and $a_{3}=-18$.

\subsubsection{Mathematical Model of Electromechanical Damping} and Onset of Galloping. Load resistance makes the damping and natural frequency of the electromechanical system be different from that without the electromechanical circuit. The impact of variations in both the frequency and damping on the system can be evaluated by analyzing the linear part of the electromechanical coupled equations. Considering the first term of the aerodynamic force $C_{y}$ only, the equation group can be rewritten as the following formation of first-order differential equations:

$$
\begin{aligned}
& \dot{X}_{1}=X_{2} \\
& \dot{X}_{2}=-\left(2 \xi \omega_{n}-\frac{\rho U^{2} D a_{1}}{2 m}\right) X_{2}-\omega_{n}{ }^{2} X_{1}+\frac{\theta}{m X_{3}}, \\
& \dot{X}_{3}=-\frac{1}{R c_{p} X_{3}}-\frac{\theta}{c_{p} X_{2}},
\end{aligned}
$$

where $X_{1}=y, X_{2}=\dot{y}$, and $X_{3}=V$. 
Equation (17) can be expressed as a vector form:

$$
\dot{X}=G(R) X,
$$

where

$$
\begin{aligned}
& X=\left(\begin{array}{l}
X_{1} \\
X_{2} \\
X_{3}
\end{array}\right), \\
& G(R) X \\
&=\left(\begin{array}{ccc}
0 & 1 & 0 \\
-\omega_{n}^{2}-\left(2 \xi \omega_{n}-\frac{\rho U^{2} D a_{1}}{2 m}\right) & \frac{\theta}{m} \\
0 & -\frac{\theta}{c_{p}} & -\frac{1}{R c_{p}}
\end{array}\right) .
\end{aligned}
$$

$$
P(t)=\left(\frac{e^{-t / R c_{p}} \theta \omega Y_{\max } R-\theta \omega Y_{\max } R\left(\cos (\omega t+\varphi)+\omega \sin (\omega t+\varphi) c_{p} R\right)}{1+\omega^{2} R^{2} c_{p}{ }^{2}}\right)^{2} R^{-1}
$$

where $P(t)$ is the power output of the system, $\omega$ is the vibrational angular frequency, and $\varphi$ is phase angle.

\subsubsection{The Validation of the Solver for Fluid-Structure Inter-} action. The fluid-structure interaction solver of OpenFOAM is verified by comparing the vibrational displacement of the square cylinder with that of Blevins [7]. Two square cylinders with a fixed length-width ratio of 2 and different damping ratios are investigated. The reduced velocity is set to 4.0 to 16.0. The computation of synchronization phenomenon considering the critical wind speed and the instable galloping response is emphatically investigated. The results are shown in Figure 4. $A / D$ refers to the nondimensional vibrational amplitude. Blevins [7] showed that the onset of synchronization of the square cylinder occurred when $U_{r}$ varied in the range of 5.0 to 5.5, and the onset of galloping $U_{r}$ was ranged between 11.0 and 11.5. The predicted critical velocities of synchronization and galloping here agree well with the work of Blevins.

The analysis of energy harvesting in electromechanical coupling of the system consists of two parts, open-circuit and close-circuit, which are identified by the load resistance. And for the open- and close-circuit system, the vortex-induced vibration (VIV) and the galloping phenomenon are both considered. The scope of reduced velocity $U_{r}$ ranges from 3.0 to 20.0 .

\section{Results of Open-Circuit System}

3.1. Energy Harvesting from the "Lock-In" Phenomenon of VIV in Open-Circuit System. Under the low wind speed the synchronization phenomenon of a square cylinder can be
The matrix $G(R) X$ has three different eigenvalues $\lambda_{i}$, $i=1,2,3$. Barrero-Gil et al. [19] indicated that the first two eigenvalues are similar to those of a pure galloping problem in the absence of the piezoelectric load, and the third eigenvalue is the result of the effect of electromechanical coupling. It is noted that the first two eigenvalues are conjugates $\left(\lambda_{2}=\right.$ $\left.\bar{\lambda}_{1}\right)$. The real and imaginary parts, respectively, represent the damping coefficient and the global frequency of the coupling system. One property of a matrix is that the product of the eigenvalues equals the value of the determinant and here it can be presented as $\lambda_{1} \lambda_{2} \lambda_{3}=-\omega_{n}{ }^{2} / R c_{p}$. The first two eigenvalues are complex conjugates; thus the value of $\lambda_{1} \lambda_{2}$ is always nonnegative; thus $\lambda_{3}$ is always real negative and the stability of the trivial solutions depends only on the first two eigenvalues. If the real part of $\lambda_{1}$ is negative, the system is stable; on the other hand, the system is unstable if the value is positive. As a result, the critical speed $U_{g}$ at $\lambda_{1}=$ 0 corresponds to the onset of instability or galloping. The electromechanical coupling power output can be obtained: obtained. Galloping could not happen when Reynolds number is lower than 159 [26]. However, the synchronization phenomenon can be effectively used to harvest energy. Figure 5 shows the computational results of vibrational amplitudes, lift coefficient, and the open-circuit voltage under low Reynolds numbers in an open-loop circuit system $(R=\infty)$.

The phenomenon synchronization can be divided into three branches: presynchronization, synchronization, and postsynchronization. In presynchronization part, the amplitude grows in a stable law. As shown in Figures 5(a), 5(b), and 5(c), when vortex shedding frequency is getting close to the natural frequency of the cylinder, the phenomenon "flap" can be clearly captured. During this period, it is worth noting that there were two major dominant frequencies in this region. However, to make a difference with the curves shown in Figures 5(g), 5(h), and 5(i), the dominant frequency in current presynchronization branch is the vortex shedding frequency shown in Figures 5(d), 5(e), and 5(f); the selfexcited vibration gets into the branch of lock-in. In this phase, the predicted amplitude quickly gets up to the stable peak value of $0.48 D$ and vortex shedding frequency is locked at the natural frequency of square cylinder.

It is worth noting that considerable oscillating energy in this branch is available for energy harvesting process, and the output open-circuit voltage can reach $5.8 \mathrm{~V}$. When $U_{r}$ is equal to 5.8 , the synchronization phenomenon bifurcates, and a high level of modulations is observed in the time histories of transverse displacement and open-circuit output voltage output, as shown in Figures 5(g), 5(h), and 5(i). The "flap" phenomenon occurs again in the branch of postsynchronization. The amplitude reduces regularly and the dominant frequency switches to the natural frequency. Modulations 


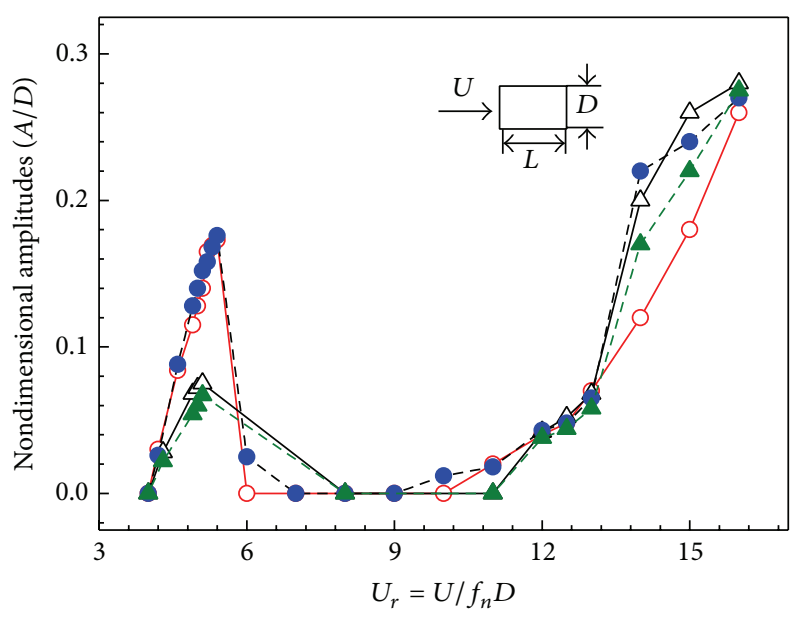

Damping ratio $\xi$

- $-0.37 \%$ Blevins (1990) - $-0.37 \%$ current work

$\triangle \quad 0.76 \%$ Blevins (1990) $\quad-\Delta-0.76 \%$ current work

FIGURE 4: Comparison of displacement of self-excited vibration in present work to previous work of Blevins under different damping ratio.

also occur in the time history of lift coefficient, as shown in Figure 5(h). From all the predicted results, the time histories of transverse displacement and output open-circuit voltage output have the same trend. This is expected; according to the Gauss law, the voltage is directly related to transverse displacement. Figures 6(a)-6(d) indicate that the vorticity contours of this instance and two different locations of the cylinder are identified by the offset of $y= \pm 0.48 D$ to its initial position. A " $2 S$ " mode vortex street is clearly shown in the region behind the square cylinder.

Variations of transverse displacement and voltage output are shown in Figure 7. It can be seen that, in the open-circuit system, the curves of transverse displacement and voltage output have three branches: presynchronization, synchronization, and postsynchronization. The best operating condition is at $U_{r}=5.6$ and in current configuration, which means that the relative wind velocity is equal to $0.39 \mathrm{~m} / \mathrm{s}$ under the parameters in present work. Therefore, the energy harvesting can be achieved from the synchronization phenomenon, even at low reduced velocity in open-circuit system.

As shown in Figure 7, the system becomes instable again when flow velocity is higher at the end of the vortex-induced vibration branch, and galloping occurs when flow velocity rises to the critical value of the instability. The amplitude gets up to a higher level while the oscillating frequency is in a lower level. From the figure it is shown that the vortexinduced vibration ends at $U_{r}=6.4$.

Meanwhile, the oscillating becomes intensive at $U_{r}=9.4$. Figure 8 reflects the time history of transverse displacement at $U_{r}=10$, the curve seems to be not as smooth as the synchronization, and the vibrational amplitude is much larger. It can be concluded that the onset velocity of galloping is up with the resistance of the system.
3.2. Effects of the Electrical Load Resistance on the Onset of Galloping. Figure 9(a) shows the variations of the real and imaginary part of the eigenvalues $\lambda_{i}$ of the linear system. It can be seen that the electromechanical damping coefficient remains low in the presence of low load resistance values. Particularly, the phenomenon resistive shunt damping effect makes the coupled electromechanical damping close to a maximum value of 0.33 . However, the natural frequency remains stable at $42.3 \mathrm{rad} / \mathrm{s}$ before $R=1.8 E 4 \Omega$, which is referred to short global frequency. For a higher $R$, the natural frequency rises up to $45.2 \mathrm{rad} / \mathrm{s}$. This value of the natural frequency is referred to the open global frequency. The curve shows that load resistance can impact the natural frequency and damping ratio of an electromechanical system. Figure 9(b) shows the effect of the electrical load resistance on the onset of the galloping. It can be seen that the onset of the galloping initially remains unchanged at $U_{r}=7.5$ when $R$ is $100 \Omega$ and increases with the enhancement of load resistance. The value gets to the peak value at $R=8 E 4 \Omega$ and decreases if the load resistance keeps increasing.

\section{Results of Close-Circuit System}

4.1. Energy Harvested from VIV in Close-Circuit System. Numerical simulations are performed by utilizing proposed damping coefficient $C$ and global frequency $\omega_{n}$. The computational results of transverse displacement and output voltage are shown in Figures 10(a)-10(d). It is clear that when $U_{r}$ falls into the range of 5.345 to 6.359 , the self-excited vibration of the square cylinder spans presynchronization, synchronization, and postsynchronization. Figure 10(a) represents the energy harvesting system with the load resistance in the aforementioned velocity range. The synchronization phenomenon begins at about the same velocity $\left(U_{r}=5.345\right)$ for all load resistances due to the fact that the global natural frequency roughly remains unchanged with the load resistance (in Figure 9(a)). It is worth noting that the amplitude of transverse displacement significantly varies with the load resistance. The maximum value of oscillation amplitude is $y / D=0.396$ when $R=100 \Omega$ and decreases to 0.348 when $R=1 E 3 \Omega$ and to 0.335 when $R=1 E 4 \Omega$. For $R=1 E 5 \Omega$, the amplitude comes to the minimum value due to the fact that the electromechanical coupling damping reaches maximum when $R=1 E 5 \Omega$ as shown in Figure 9(a).

On the other hand, Figure 10(a) shows that the width of the synchronization region is approximately unchanged with electromechanical coupling damping caused by load resistance. Variation trends of the root mean square closecircuit voltage output in Figures 10(b) and 10(c) are shown in two different forms. Figure 10(b) shows the variation trend of voltage output by the reduced velocity under different load resistance. The voltage output gets higher with the increasing of the load resistance; the maximum is attained when $U_{r}=$ 5.78 and $R=1 E 6 \Omega$. When $R=1 E 5 \Omega$ and $U_{r}=5.96$, the maximum voltage output is about $0.288 \mathrm{~V}$, and when $R=1 \mathrm{~K} \Omega$ and $U_{r}=6.05$, the maximum decreases to $0.023 \mathrm{~V}$. When $R=100 \Omega$, the voltage output is lower and the maximum value is $1.81 E-3 \mathrm{~V}$. It can be concluded 


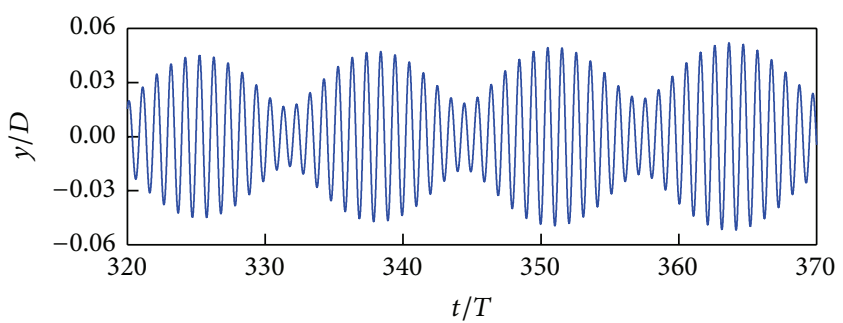

(a)

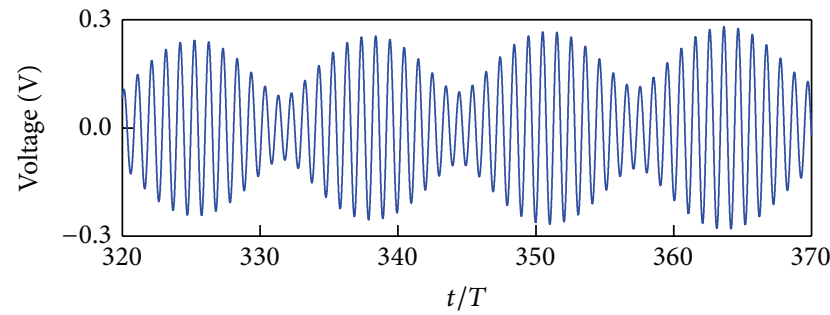

(c)

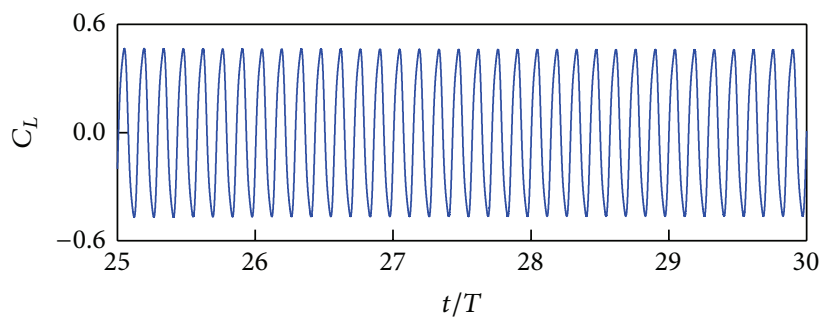

(e)

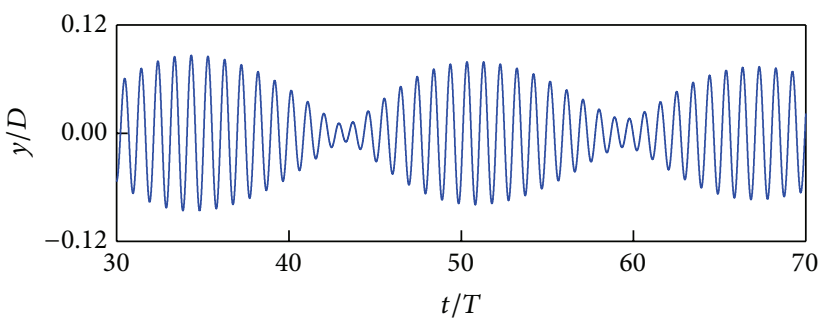

(g)

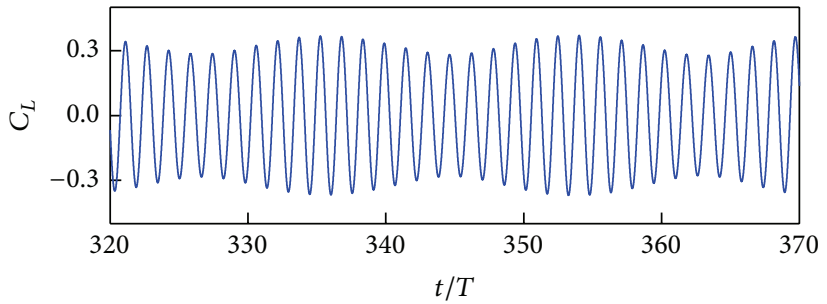

(b)

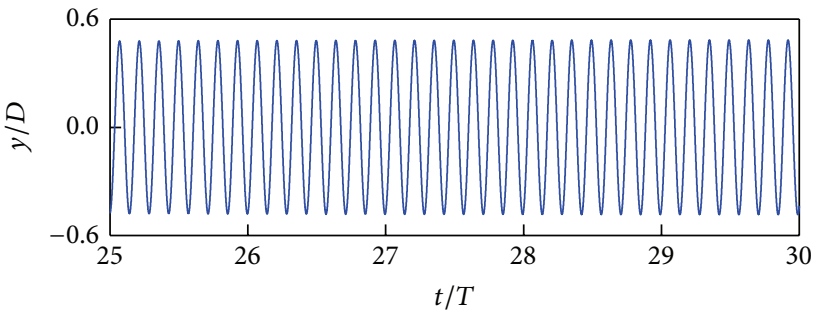

(d)

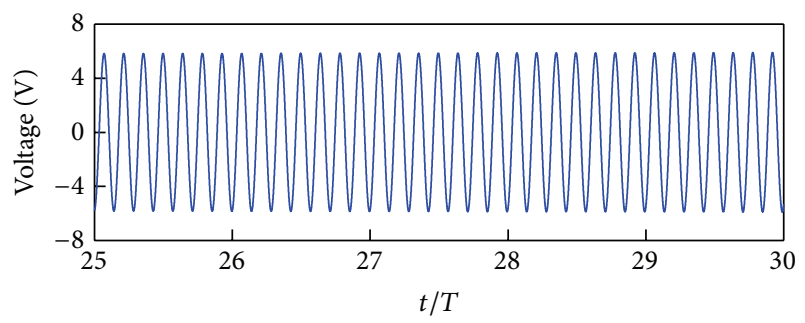

(f)

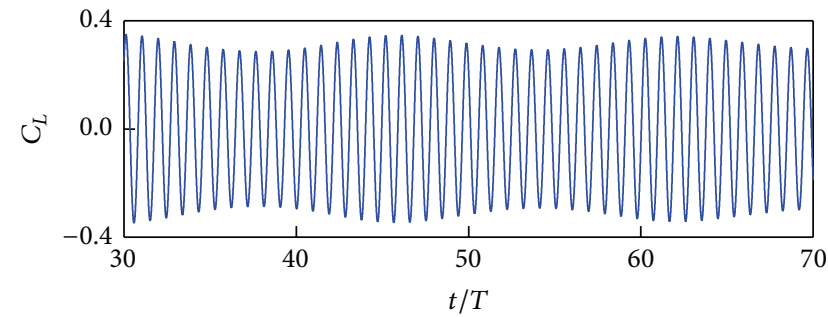

(h)

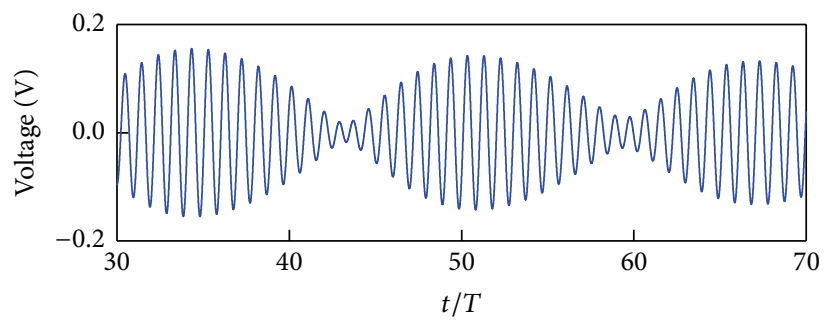

(i)

FIGURE 5: Time histories of transverse displacement, lift, and output voltage for regime of presynchronization (a, b, c); synchronization (d, e, f); postsynchronization (g, h, i) in the open-circuit system.

that the voltage output of the close-circuit system increases with the increasing of load resistance. This conclusion can also be obtained from Figure 10(c), which shows the variations of voltage output with load resistance under different flow velocities. As the load resistance increases, the voltage increases in a stable law. Figure 10(d) shows variations of harvested power with flow velocity under different load resistance. The harvested power is computed with the voltage according to following equation:

$$
P_{\text {har }}=\frac{V^{2} \text { rms }}{R},
$$




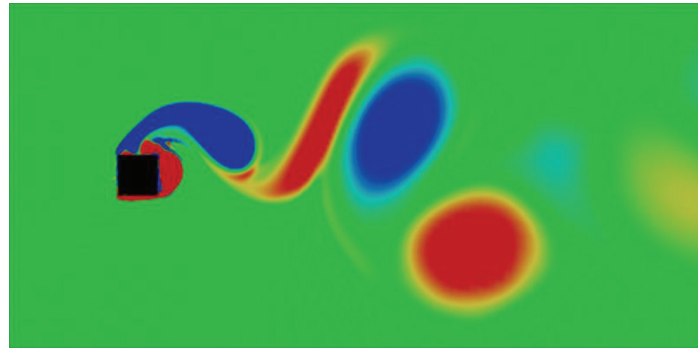

(a)

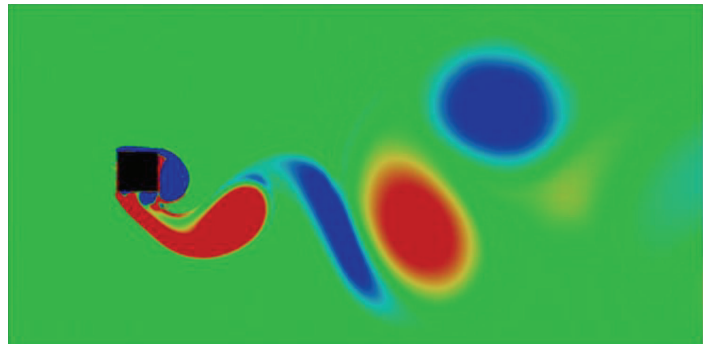

(c)

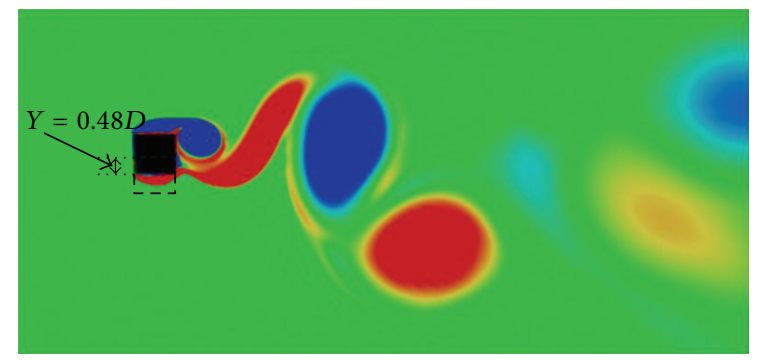

(b)

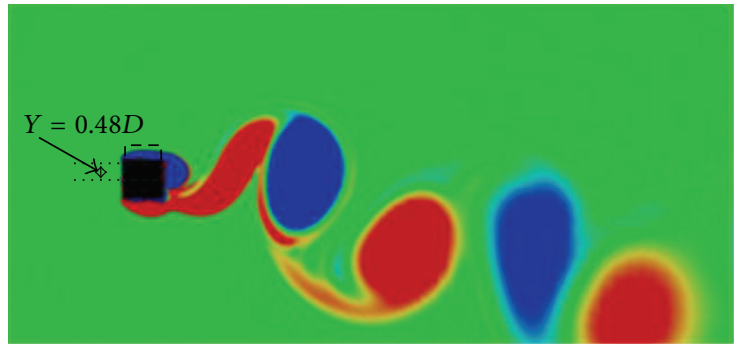

(d)

FIGURE 6: Vorticity contours of the vortex street: (a) the original position of the cylinder; (b) the cylinder moving to the top position in the flow; (c) the cylinder moving back to the original position; (d) the cylinder moving to the bottom position in the flow.

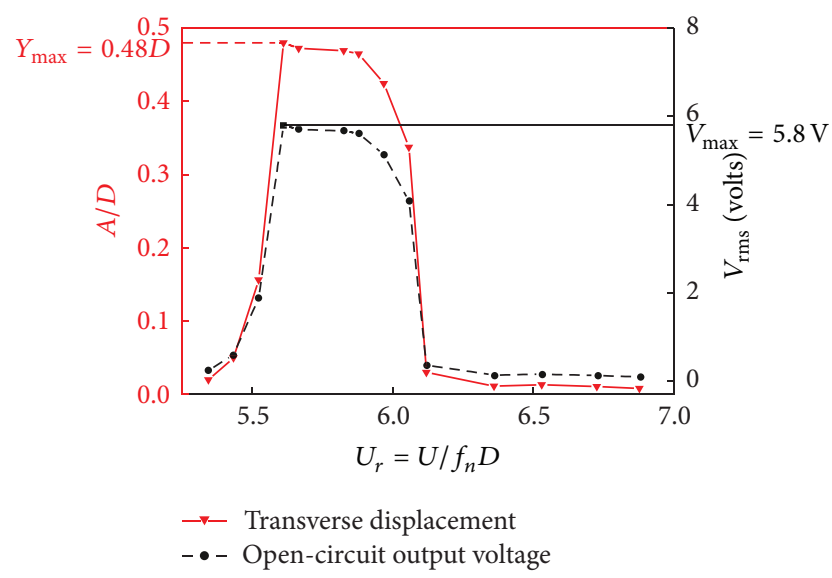

FiguRE 7: Variation trends of the transverse displacement and output voltage of the vortex-induced vibration of open-circuit system.

where $V_{\text {rms }}$ indicates the root mean square value or the effective value of the voltage output and $R$ represents the load resistance across the piezoelectric transducer. Figure 10(d) shows that the maximum harvested power can be obtained under all considered $R$ in the synchronization system when $R=1 E 6 \Omega$. The harvested power reaches its maximum value, $P_{\text {har }}=1.14 E-5 \mathrm{~W}$. A notable phenomenon is that, for all considered $R$, the harvested power can also be enhanced with the increase of $R$.

4.2. Energy Harvesting Resulted from Galloping in the CloseCircuit System. To analyze the nonlinear problem of the electromechanical coupling system, the governing equations are directed computed on OpenFOAM. Following the synchronization branch, the galloping phenomenon occurs with higher flow velocities. The transverse displacement can reach a more high level than that of synchronization branch; thus more energy can be harvested. However, the oscillating frequency of galloping is much lower than that of synchronization.

Figure 11 shows the transverse displacement, voltage output, and harvested energy under different $R$ caused by different reduced velocities of galloping of the square cylinder. The load resistance varies with the onset speed of galloping. In Figure 11(a), the transverse displacement rises with the increase of free stream velocity. The onset speed reaches its maximum value when $R=2 E 5 \Omega$; the result also shows that the load resistance contributes to variations of the amplitude of the transverse displacement. The transverse displacement has the smallest value when $R=2 E 5 \Omega$, for the electromechanical damping is in the highest level. However, the voltage output could not be accompanied with an increase in the harvested power, as shown in Figure 11(b). It is worth noting that there is an optimum value of load resistance for maximizing the harvested power. The maximum level is obtained when $R=2 E 5 \Omega$. Under this selected load resistance, the harvested power is nearly 1.4 watts with the reduced velocity of 20.0 or so.

\section{Conclusions}

In present work, energy harvesting from both vortex-induced vibration and galloping phenomenon from SEV of a square cylinder has been investigated under different wind speeds in platform of OpenFOAM. The analysis of electromechanical coupling system is carried out under both open- and close-circuit parts. In open-circuit system, three branches 


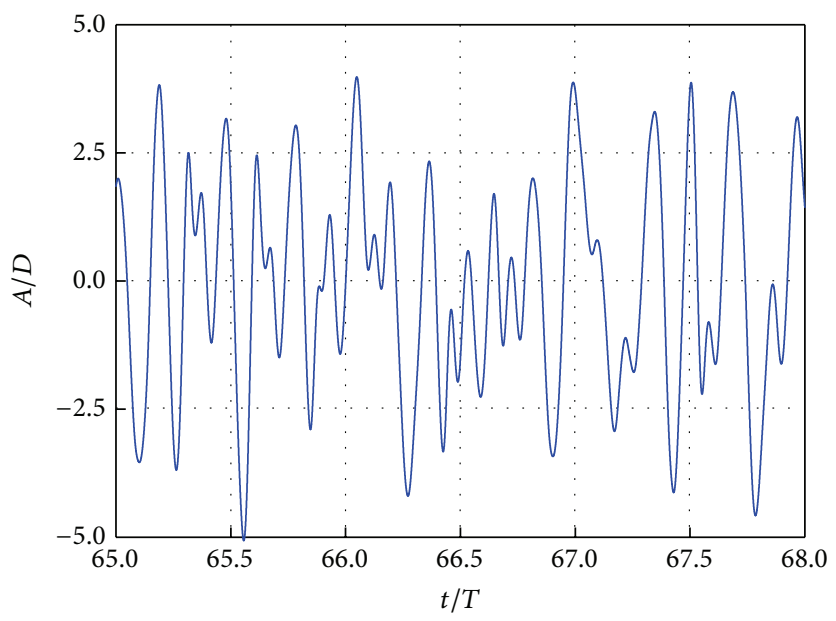

FIGURE 8: Time history of transverse displacement of galloping of the square cylinder at $U_{r}=10.0$.

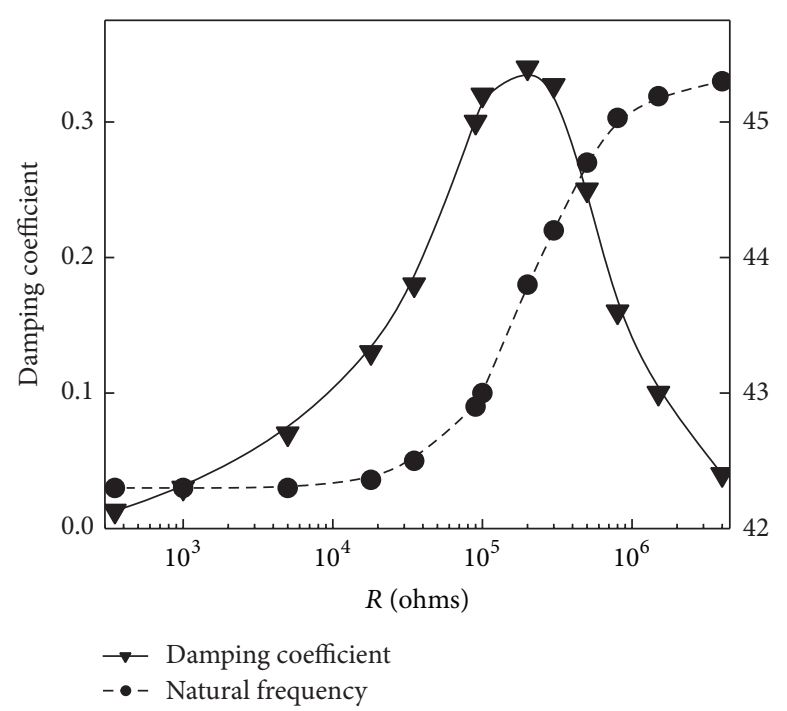

(a)

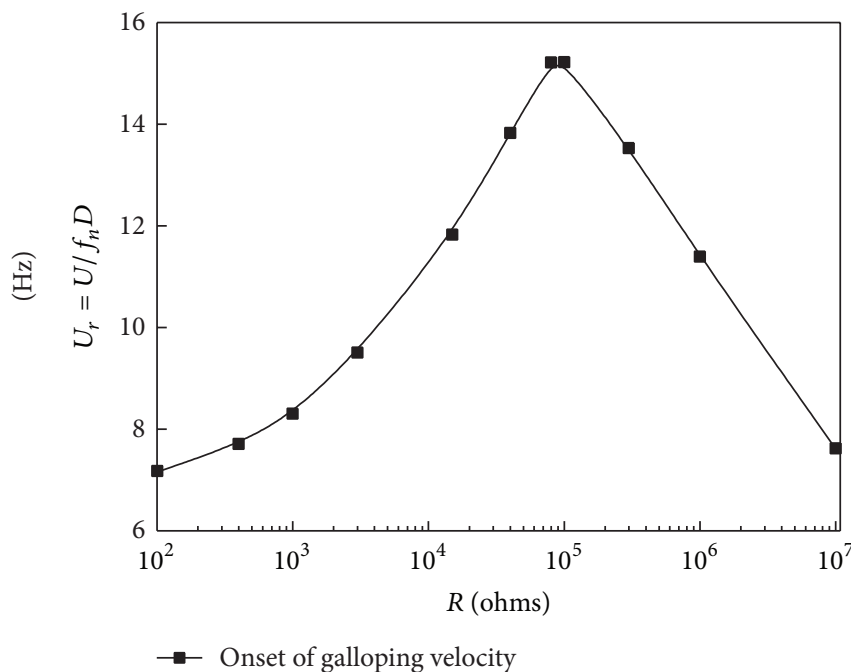

(b)

FIGURE 9: (a) Variations of damping coefficient and natural frequency of the linear system with the load resistance. (b) Variations of galloping velocity with the load resistance.

in vortex-induced vibration have been obtained and the vibrational displacement of both VIV and galloping has been investigated. It can be concluded that in VIV the vibrational displacement curves were varying in a sinusoidal law which is different from the galloping regime. The vortex shedding pattern has been shown by vortex contours. The max value of open-circuit voltage output in VIV regime has been obtained to $5.8 \mathrm{~V}$ at $U_{r}=5.8$ in synchronization regime. For closecircuit system, the effects of load resistance on the natural frequency and system damping have been analyzed; the harvested power and transverse displacement of the square cylinder are investigated. For VIV, the maximum harvested power reached maximum $P_{\text {har }}=1.14 E-5 \mathrm{~W}$ when $R=$ $1 E 6 \Omega$. For galloping, the harvested power is $1.4 \mathrm{~W}$ when $R=2 E 5 \Omega$. It can be concluded that synchronization phenomenon can be utilized to harvest power when the velocity of wind is low. When wind speed gets higher to galloping, the open-circuit voltage output increases as the wind speed increases. In close-circuit system, the harvested power can be enhanced by increasing load resistance in the low wind speed region. For high wind speed applications, the harvested power can be optimized to obtain the minimum of oscillating displacement by choosing adaptive load resistance.

\section{Nomenclature}

$U:$ Flow velocity $(\mathrm{m} / \mathrm{s})$

$D$ : Characteristic length (m)

$y$ : Transverse displacement (m)

$M$ : Mass $(\mathrm{Kg})$

$K$ : Stiffness $(\mathrm{N} / \mathrm{m})$

$C$ : Damping $(\mathrm{Nm} / \mathrm{s})$ 


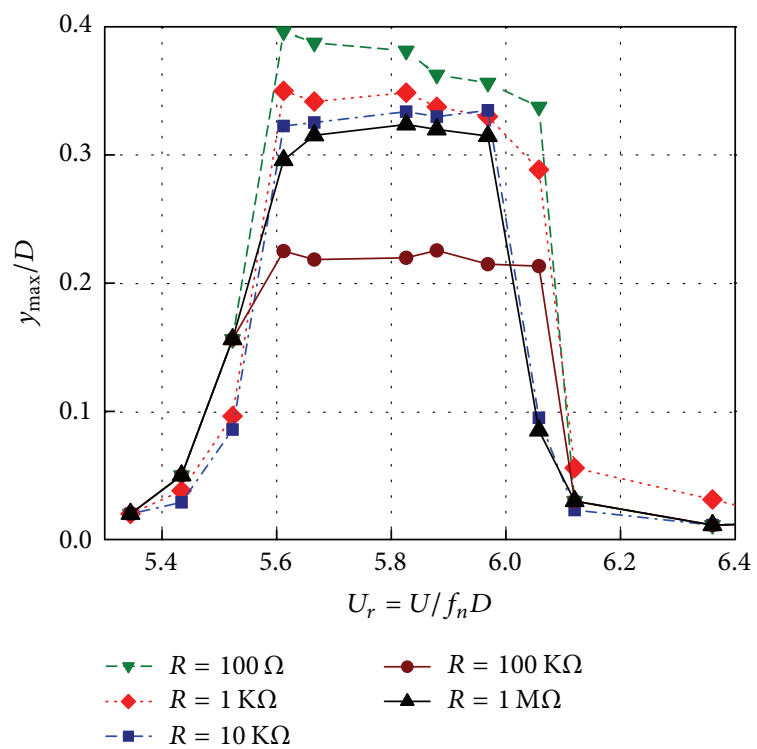

(a)

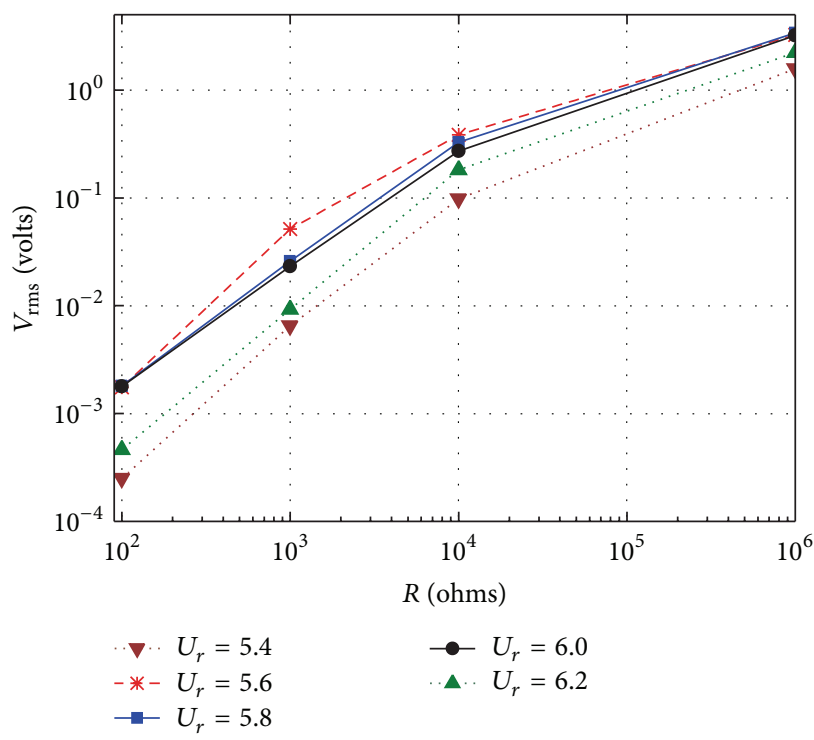

(c)

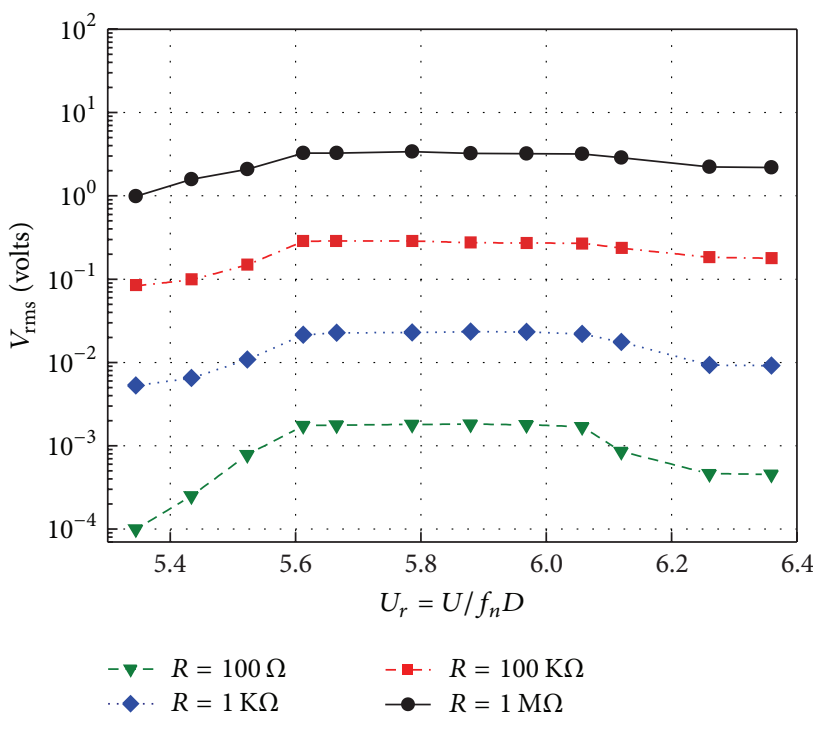

(b)

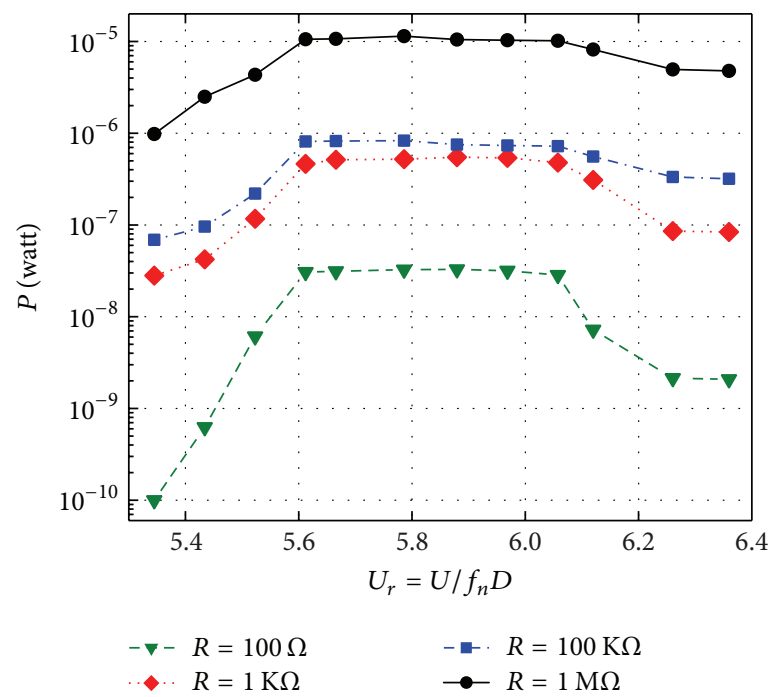

(d)

Figure 10: Variations of (a) transverse displacement; ((b) and (c)) voltage output; (d) harvested power with the nondimensional flow velocity.

$f:$ Frequency $(\mathrm{Hz})$

$R$ : Load resistance (ohms)

F: Force $(\mathrm{N})$

Re: Reynolds number

$P$ : Pressure $(\mathrm{Pa})$

$\omega$ : Circular frequency $(\mathrm{Hz})$

$\xi$ : Damping coefficient

$\theta$ : Electromechanical coupling (N/V)

$\tau: \quad$ Stress $(\mathrm{N})$

$\Omega$ : Deforming wet surface

$\lambda_{i}$ : Eigenvalues

$U_{r}$ : Reduced velocity

$f_{n}$ : Natural frequency
$P_{\text {har: }}$ Harnessed power

$U_{\mathrm{rms}}$ : Root mean square voltage.

\section{Competing Interests}

All the funds do not lead to any competing interests regarding the publication of this paper.

\section{Acknowledgments}

This work was supported by Specialized Research Fund for the Natural Science Foundation of Guangdong Province (Grant no. 2015A030310182), Open Fund of Chongqing 


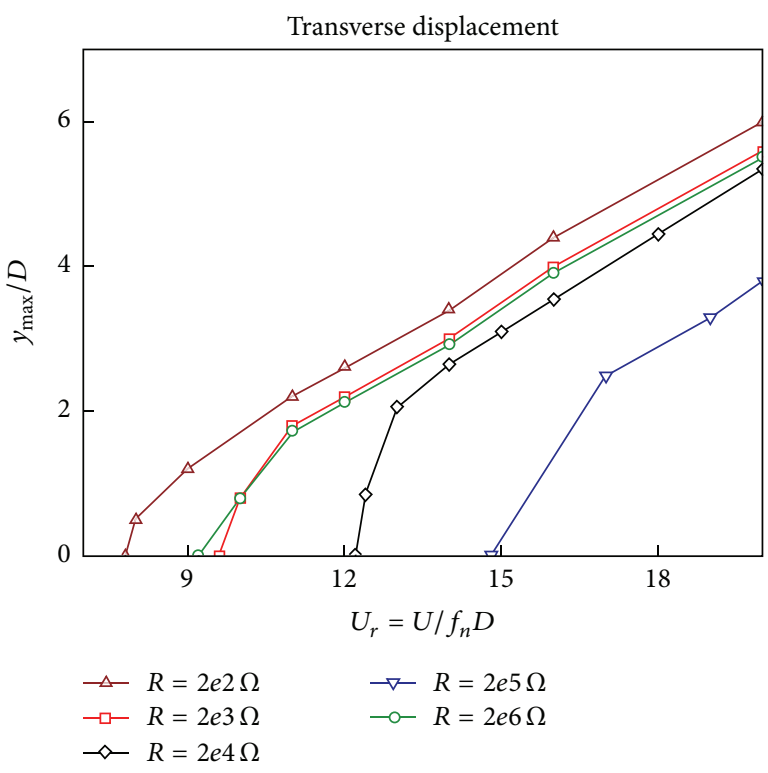

(a)

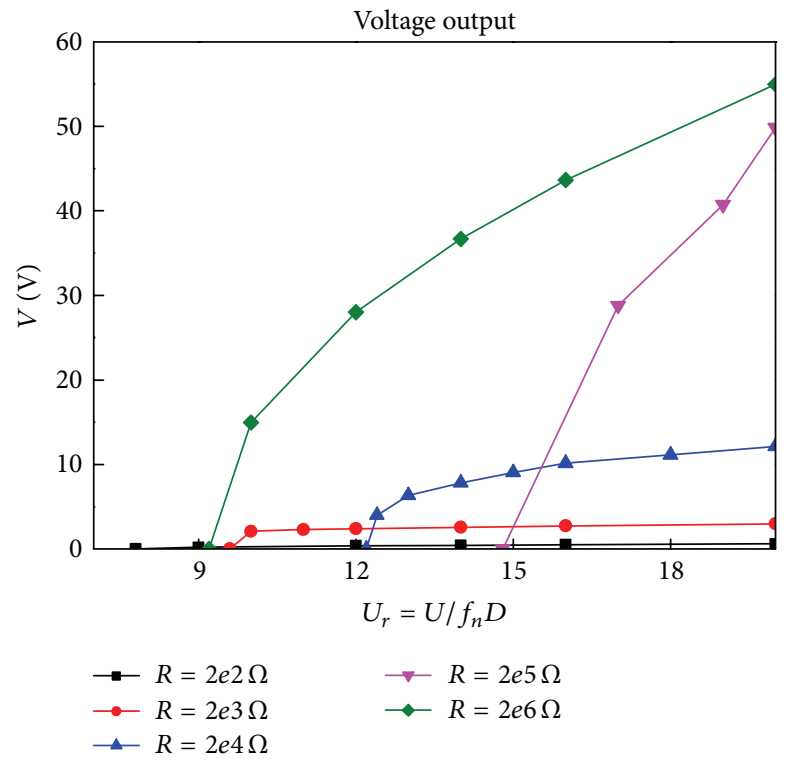

(b)

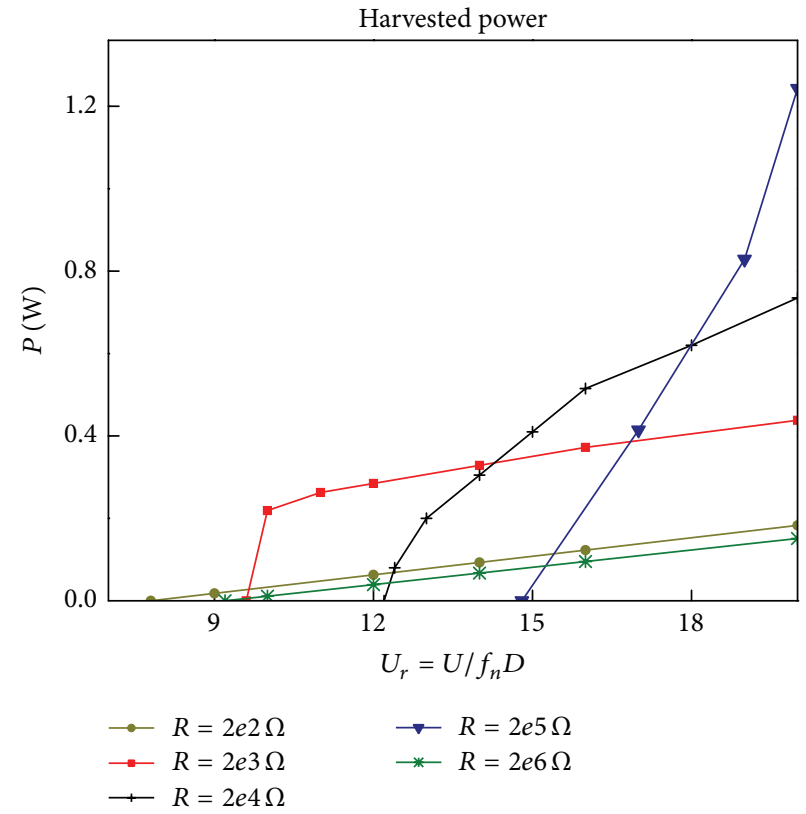

(c)

FIGURE 11: Variations of (a) transverse displacement and (b) voltage output (c) harvested power with the nondimensional flow velocity.

University, Key Laboratory of Low-Grade Energy Utilization Technologies and Systems (Grant no. LLEUTS-201610), and Open Fund of Jiangsu Engineering Research Center on Meteorological Energy Using and Control/C-MEIC, Nanjing University of Information Science \& Technology (Grant no. KCMEIC02).

\section{References}

[1] I. F. Akyildiz, W. Su, Y. Sankarasubramaniam, and E. Cayirci, "Wireless sensor networks: a survey," Computer Networks, vol. 38, no. 4, pp. 393-422, 2002.
[2] C. Alippi, R. Camplani, C. Galperti, and M. Roveri, "A robust, adaptive, solar-powered WSN framework for aquatic environmental monitoring," IEEE Sensors Journal, vol. 11, no. 1, pp. 45$55,2011$.

[3] T. Arampatzis, J. Lygeros, and S. Manesis, "A survey of applications of wireless sensors and wireless sensor networks," in Proceedings of the 20th IEEE International Symposium on Intelligent Control (ISIC '05) and the13th Mediterranean Conference on Control and Automation (MED '05), pp. 719-724, Limassol, Cyprus, June 2005.

[4] D. Brunelli, C. Moser, L. Thiele, and L. Benini, "Design of a solar-harvesting circuit for batteryless embedded systems," 
IEEE Transactions on Circuits and Systems I: Regular Papers, vol. 56, no. 11, pp. 2519-2528, 2009.

[5] W. K. G. Seah, Z. A. Eu, and H.-P. Tan, "Wireless sensor networks powered by ambient energy harvesting (WSN-HEAP) survey and challenges," in Proceedings of the 1st International Conference on Wireless Communication, Vehicular Technology, Information Theory and Aerospace \& Electronic Systems Technology (Wireless VITAE '09), pp. 1-5, Aalborg, Denmark, May 2009.

[6] G. Werner-Allen, K. Lorincz, M. Welsh et al., "Deploying a wireless sensor network on an active volcano," IEEE Internet Computing, vol. 10, no. 2, pp. 18-25, 2006.

[7] R. D. Blevins, "Flow-induced vibration," 1990.

[8] G. W. Taylor, J. R. Burns, S. M. Kammann, W. B. Powers, and T. R. Welsh, "The energy harvesting Eel: a small subsurface ocean/river power generator," IEEE Journal of Oceanic Engineering, vol. 26, no. 4, pp. 539-547, 2001.

[9] J. J. Allen and A. J. Smits, "Energy harvesting EEL," Journal of Fluids and Structures, vol. 15, no. 3-4, pp. 629-640, 2001.

[10] W. P. Robbins, I. Marusic, D. Morris, and T. O. Novak, "Windgenerated electrical energy using flexible piezoelectric mateials," in Proceedings of the ASME International Mechanical Engineering Congress and Exposition, pp. 581-590, Chicago, Ill, USA, November 2006.

[11] K. Raghavan and M. M. Bernitsas, "Experimental investigation of Reynolds number effect on vortex induced vibration of rigid circular cylinder on elastic supports," Ocean Engineering, vol. 38, no. 5-6, pp. 719-731, 2011.

[12] J. H. Lee and M. M. Bernitsas, "High-damping, high-Reynolds VIV tests for energy harnessing using the VIVACE converter," Ocean Engineering, vol. 38, no. 16, pp. 1697-1712, 2011.

[13] W. Wu, M. M. Bernitsas, and K. Maki, "RANS simulation vs. experiments of flow induced motion of circular cylinder with passive turbulence control at 35,000 < Re < 130,000," in Proceedings of the ASME 2011 30th International Conference on Ocean, Offshore and Arctic Engineering, pp. 733-744, American Society of Mechanical Engineers, Rotterdam, Netherlands, June 2011.

[14] L. Ding, M. M. Bernitsas, and E. S. Kim, “2-D URANS vs. experiments of flow induced motions of two circular cylinders in tandem with passive turbulence control for $30,000<R e<105,000$," Ocean Engineering, vol. 72, pp. 429-440, 2013.

[15] A. Mehmood, A. Abdelkefi, M. R. Hajj, A. H. Nayfeh, I. Akhtar, and A. O. Nuhait, "Piezoelectric energy harvesting from vortexinduced vibrations of circular cylinder," Journal of Sound and Vibration, vol. 332, no. 19, pp. 4656-4667, 2013.

[16] A. Abdelkefi, M. R. Hajj, and A. H. Nayfeh, "Phenomena and modeling of piezoelectric energy harvesting from freely oscillating cylinders," Nonlinear Dynamics, vol. 70, no. 2, pp. 13771388, 2012.

[17] A. Abdelkefi, M. R. Hajj, and A. H. Nayfeh, "Piezoelectric energy harvesting from transverse galloping of bluff bodies," Smart Materials and Structures, vol. 22, no. 1, Article ID 015014, 2013.

[18] A. Abdelkefi, M. R. Hajj, and A. H. Nayfeh, "Power harvesting from transverse galloping of square cylinder," Nonlinear Dynamics, vol. 70, no. 2, pp. 1355-1363, 2012.

[19] A. Barrero-Gil, G. Alonso, and A. Sanz-Andres, "Energy harvesting from transverse galloping," Journal of Sound and Vibration, vol. 329, no. 14, pp. 2873-2883, 2010.
[20] A. Abdelkefi and A. O. Nuhait, "Modeling and performance analysis of cambered wing-based piezoaeroelastic energy harvesters," Smart Materials and Structures, vol. 22, no. 9, Article ID 095029, 2013.

[21] H. L. Dai, A. Abdelkefi, and L. Wang, "Piezoelectric energy harvesting from concurrent vortex-induced vibrations and base excitations," Nonlinear Dynamics, vol. 77, no. 3, pp. 967-981, 2014.

[22] Z. Yan and A. Abdelkefi, "Nonlinear characterization of concurrent energy harvesting from galloping and base excitations," Nonlinear Dynamics, vol. 77, no. 4, pp. 1171-1189, 2014.

[23] J. P. den Hartog, Mechanical Vibrations, Dover, Mineola, NY, USA, 1956.

[24] G. Parkinson, "Mathematical models of flow-induced vibrations of bluff bodies," in Flow-Induced Structural Vibrations, pp. 81-127, Springer, Berlin, Germany, 1974.

[25] G. V. Parkinson and J. D. Smith, "The square prism as an aeroelastic non-linear oscillator," The Quarterly Journal of Mechanics and Applied Mathematics, vol. 17, no. 2, pp. 225-239, 1964.

[26] A. Barrero-Gil, A. Sanz-Andrés, and G. Alonso, "Hysteresis in transverse galloping: the role of the inflection points," Journal of Fluids and Structures, vol. 25, no. 6, pp. 1007-1020, 2009. 


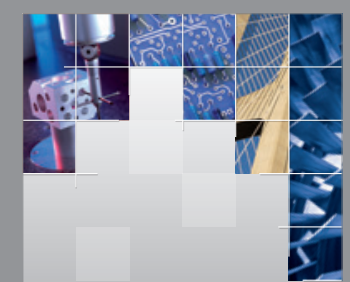

\section{Enfincering}
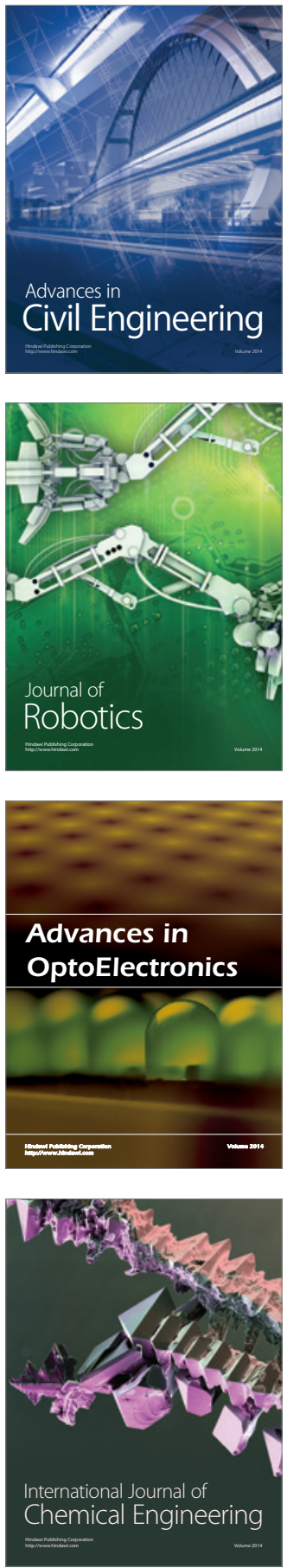

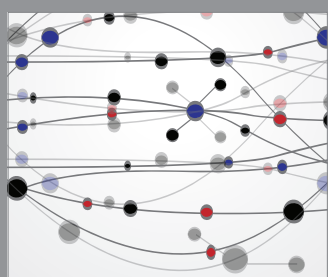

The Scientific World Journal

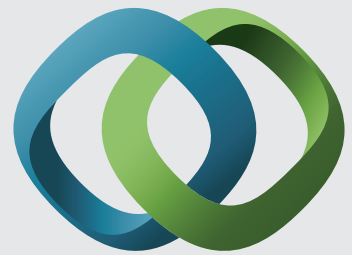

\section{Hindawi}

Submit your manuscripts at

http://www.hindawi.com
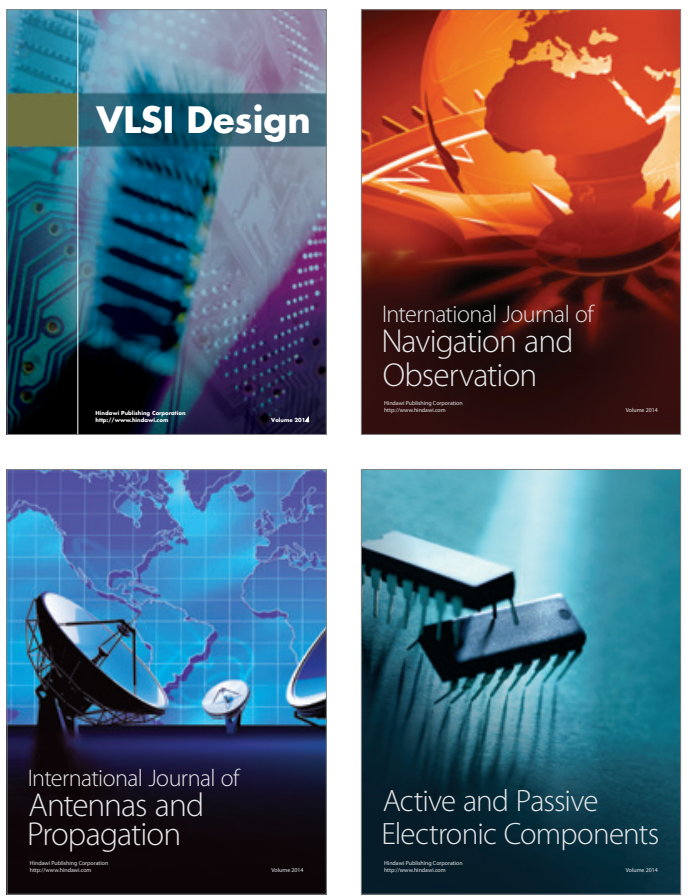
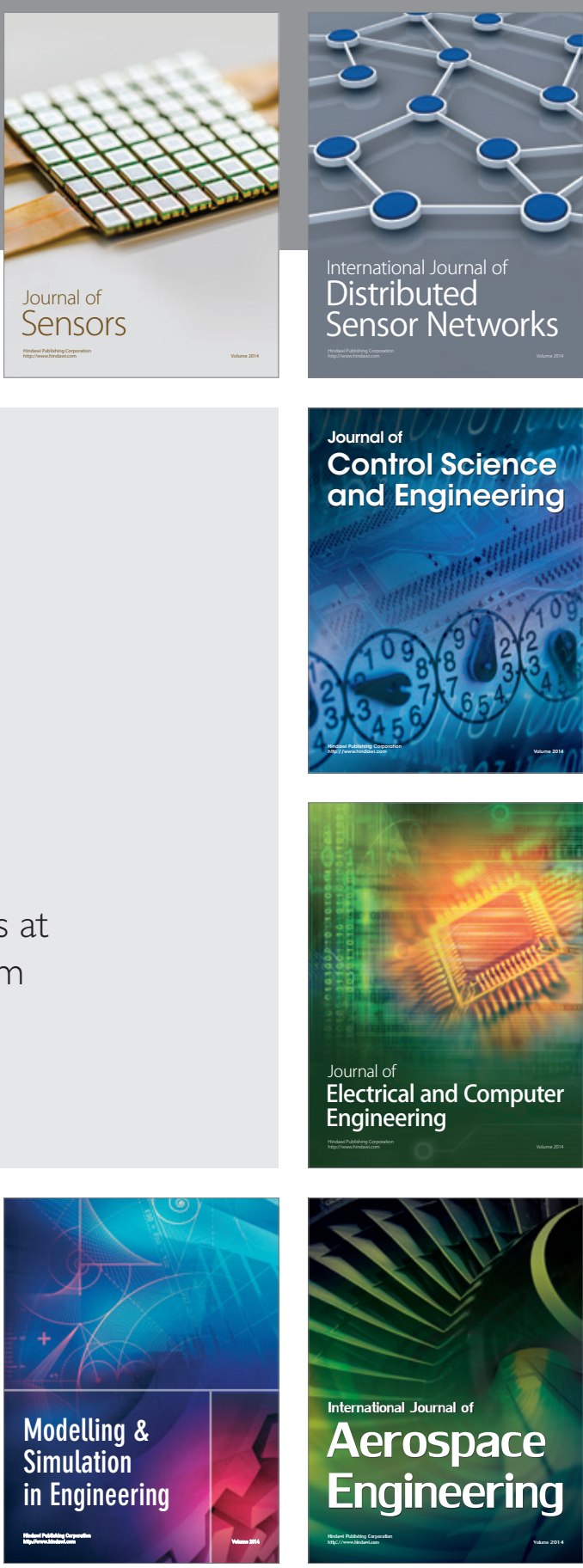

International Journal of

Distributed

Sensor Networks

Journal of

Control Science

and Engineering
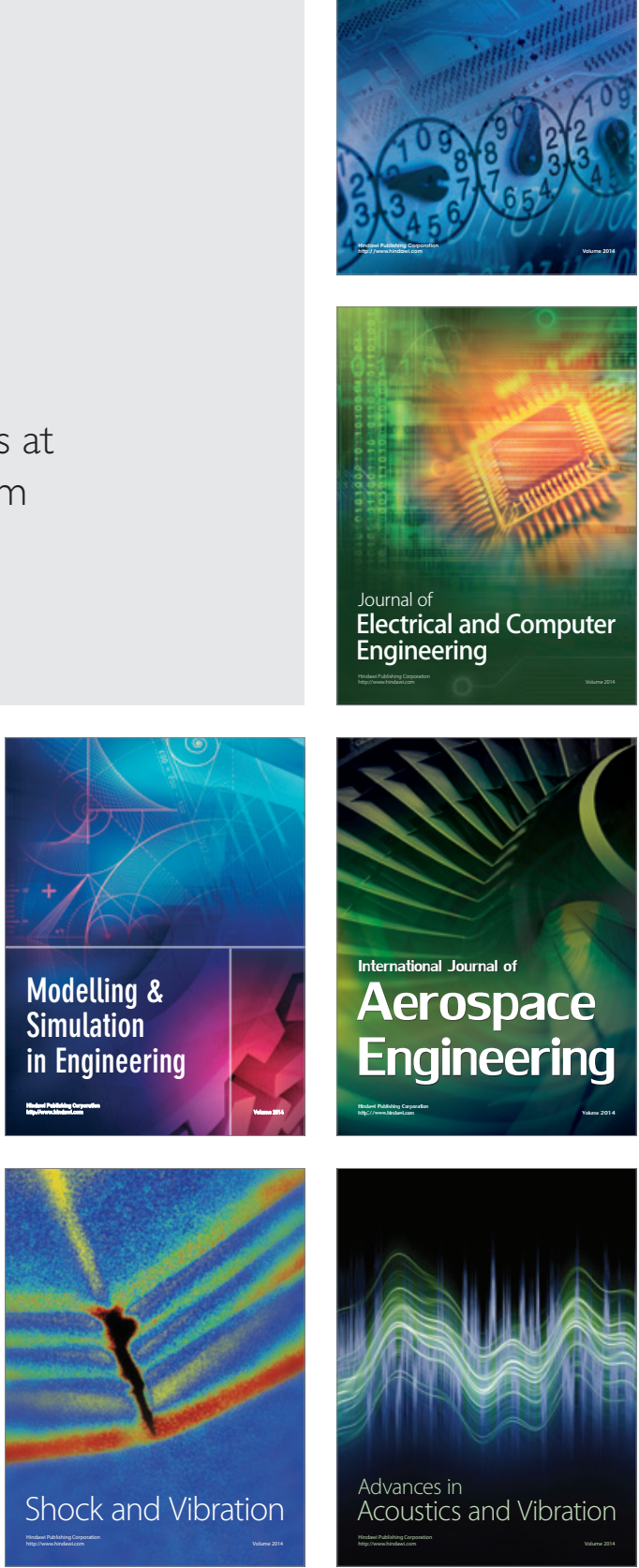